Pacific Journal of Mathematics

WAVE OPERATORS AND UNITARY EQUIVALENCE 


\section{WAVE OPERATORS AND UNITARY EQUIVALENCE}

\section{Tosio Kato}

This paper is concerned with the wave operators $W_{ \pm}=$ $W_{ \pm}\left(H_{1}, H_{0}\right)$ associated with a pair $H_{0}, H_{1}$ of selfadjoint operators. Let $(M)$ be the 'set of all real-valued functions $\phi$ on reals such that the interval $(-\infty, \infty)$ has a partition into a finite number of open intervals $I_{k}$ and their end points with the following properties: on each $I_{k}, \phi$ is continuously differentiable, $\phi^{\prime} \neq 0$ and $\phi^{\prime}$ is locally of bounded variation. Theorem 1 states that, if $H_{1}=H_{0}+V$ where $V$ is in the trace class $\boldsymbol{T}$, then $W^{\prime}{ }_{ \pm} \pm W_{ \pm}\left(\phi\left(H_{1}\right), \phi\left(H_{0}\right)\right)$ exist and are complete for any $\phi \in(M)$; moreover, $M^{\prime}{ }_{ \pm}$are "piecewise equal" to $W_{ \pm}$ (in the sense to be specified in text). Theorem 2 strengthens Theorem 1 by replacing the above assumption by the condition that $\phi_{n}\left(H_{1}\right)=\phi_{n}\left(H_{0}\right)+V_{n}, V_{n} \in T$, where $\phi_{n} \in(M)$ and $\phi_{n}$ is univalent on $(-n, n)$ for $n=1,2,3, \ldots$ As corollaries we obtain many useful sufficient conditions for the existence and completeness of wave operators.

1. Introduction. The present paper is a continuation of earlier papers of the author on the theory of wave and scattering operators and the related theory of unitary equivalence of selfadjoint operators.

We begin with a brief review of relevant definitions and known results (see Kato [4, 5] and Kuroda [6]), adding some new definitions for convenience. Let $\mathfrak{S}$ be a Hilbert space and let $H$ be a selfadjoint operator in $\mathfrak{S}$ with the spectral representation $H=\int \lambda d E(\lambda)$. A vector $u \in \mathfrak{K}$ is absolutely continuous (singular) with respect to $H$ if $(E(\lambda) u, u)$ is absolutely continuous (singular) in $\lambda$ (with respect to the Lebesgue measure). The set of all $u \in \mathfrak{S}$ which are absolutely continuous (singular) with respect to $H$ forms a subspace of $\mathfrak{S}$, which we call the absolutely continuous (singular) subspace with respect to $H$ and denote by $\mathfrak{S}_{a c}\left(\mathfrak{S}_{s}\right)$. These two subspaces are orthogonal complements to each other and reduce $H$. The part of $H$ in $\mathfrak{S}_{a c}\left(\mathfrak{S}_{s}\right)$ is called the absolutely continuous (singular) part of $H$ and is denoted by $H_{a c}\left(H_{s}\right)$

Let $H_{j}, j=0,1$, be two selfadjoint operators in $\mathfrak{L}$ with the spectral representation $H_{j}=\int \lambda d E_{j}(\lambda)$, and let $P_{j}$ be the projection on the absolutely continuous subspace $\mathfrak{S}_{j, a c}$ with respect to $H_{j}$. If one or both of the strong limits

$$
W_{ \pm}=W_{ \pm}\left(H_{1}, H_{0}\right)=s-\lim _{t \rightarrow \pm \infty} \exp \left(i t H_{1}\right) \exp \left(-i t H_{0}\right) P_{0}
$$

exist(s), it is (they are) called the (generalized) wave operator(s).

Received January 8, 1964. This work was sponsored (in part) by Office of Naval Research Contract 222 (62). 
$W_{+}$is, whenever it exists, a partial isometry on $\mathfrak{S}$ with initial set $\mathfrak{S}_{0, a c}$ and final set $\mathfrak{M}_{+}$contained in $\mathfrak{K}_{1, a c}$. $\mathfrak{M}_{+}$reduces $H_{1}$, and the part of $H_{1}$ in $\mathfrak{M}_{+}$is unitarily equivalent to $H_{0, a c}$, with

$$
E_{1}(\lambda) W_{+}=W_{+} E_{0}(\lambda),
$$$$
-\infty<\lambda<+\infty
$$

The wave operator $W_{+}$will be said to be complete if the final set $\mathfrak{M}_{+}$ coincides with $\mathfrak{S}_{1, a c}$.

$W_{+}$has the property that, whenever $W_{+}\left(H_{1}, H_{0}\right)$ and $W_{+}\left(H_{2}, H_{1}\right)$ exist, then $W_{+}\left(H_{2}, H_{0}\right)$ exists and is equal to $W_{+}\left(H_{2}, H_{1}\right) W_{+}\left(H_{1}, H_{0}\right)$. If both $W_{+}\left(H_{1}, H_{0}\right)$ and $W_{+}\left(H_{0}, H_{1}\right)$ exist, then they are complete and are the adjoints to each other.

Similar results hold for $W_{+}$replaced by $W_{-}$.

If $H_{1}-H_{0}$ is small in the sense that $H_{1}=H_{0}+V$ with $V$ belonging to the trace class $\boldsymbol{T}$ of operators on $\mathfrak{S}$, then both $W_{ \pm}\left(H_{1}, H_{0}\right)$ exist and are complete. The main purpose of the present paper is to prove some generalizations of this theorem, which involve what we shall call the principle of invariance of wave operators. Roughly speaking, this principle asserts that the wave operators $W_{ \pm}\left(\phi\left(H_{1}\right), \phi\left(H_{0}\right)\right)$ exist for an "arbitrary" function $\phi$ and are independent of $\phi$ for a wide class of functions $\phi$. Its precise formulation is given in Theorems 1 and 2 proved below.

The proof of these theorems is rather simple, depending essentially on a single inequality proved in a previous paper (Kato [5]). It will be noted that Theorem 2 contains as special cases most of the sufficient conditions for the existence and completeness of wave operators obtained in recent years (see Kuroda [6, 7], Birman [1, 2], BirmanKrein [3].

2. Principle of invariance of wave operators. Consider the wave operators $W_{ \pm}\left(\phi\left(H_{1}\right),\left(\phi\left(H_{0}\right)\right)\right.$ where $\phi$ is a real-valued, Borel measurable function on $(-\infty,+\infty)$. The principle of invariance asserts that these wave operators do not denoted on $\phi$. Of course certain restrictions must be imposed on $\phi$ and on the relation between $H_{0}$ and $H_{1}$ To this end it is convenient to introduce a certain class of functions.

Definition. A real-valued function $\phi$ on $(-\infty,+\infty)$ is said to be of class $(M)$ if the whole interval $(-\infty,+\infty)$ has a partition into a finite number of open intervals $I_{k}, k=1, \cdots, r$, and their end points with the following properties: on each $I_{k}, \phi$ is strictly monotone and differentiable, with the derivative $\phi^{\prime}$ continuous, $\phi^{\prime} \neq 0$ and (locally) of bounded variation. $\left\{I_{k}\right\}$ will be called a system of intervals associated with $\phi$ (such a system is not unique).

THEOREM 1. Let $H_{0}, H_{1}$ be selfadjoint operators such that $H_{1}=$ 
$H_{0}+V$ with $V \in \boldsymbol{T}$. If $\phi$ is of class $(M), \quad{W^{\prime}}^{\prime}=W_{ \pm}\left(\phi\left(H_{1}\right), \phi\left(H_{0}\right)\right)$ exist and are complete. Furthermore, $W^{\prime}$ are "piecewise equal" either to $W_{ \pm}=W_{ \pm}\left(H_{1}, H_{0}\right)$ or to $W_{\mp}$, in the sense that

$$
\left(W^{\prime}{ }_{ \pm}-W_{ \pm}\right) E_{0}\left(I_{k}\right)=0 \text { or }\left(W^{\prime}{ }_{ \pm}-W_{\mp}\right) E_{0}\left(I_{k}\right)=0, k=1, \cdots, r,
$$

according as $\phi$ is increasing or decreasing on $I_{k}$. In particular, ${W^{\prime}}^{\prime}=W_{ \pm}\left(W^{\prime}{ }_{ \pm}=W_{\mp}\right)$ if $\phi$ is increasing (decreasing) in each $I_{k}, k=$ $1, \cdots, r$. (Here $\left\{I_{k}\right\}$ is a system of intervals associated with $\phi \in(M)$ and $E_{0}(I)=E_{0}(\beta-0)-E_{0}(\alpha)$ if $I=(\alpha, \beta)$.)

Proof. It is known (see Kato [5]) that $W_{ \pm}$exist under the assumptions of the theorem.

We take a fixed $I_{k}$ and assume that $\phi$ is increasing on $I_{k}$. We use the inequality (2.9) of the paper cited, which reduces for $s=0$ to

$$
\begin{aligned}
& \left\|\left(W_{+}-1\right) x\right\| \leqq\left(8 \pi m^{2}\|V\|_{1}\right)^{1 / 4} \\
& \quad \times\left(\int_{0}^{+\infty}\left\||V|^{1 / 2} \exp \left(-i t H_{0}\right) x\right\|^{2} d t\right)^{1 / 4},
\end{aligned}
$$

where $x \in \mathfrak{C}_{0, a c}$ is subjected to the condition that $d\left(E_{0}(\lambda) x, x\right) / d \lambda \leqq m^{2}$ almost everywhere. Here $|V|$ is the nonnegative square roof of $V^{2}$ and $\|V\|_{1}$ denotes the trace norm of $V$, which is finite by assumption.

Now let $u \in \mathfrak{S}_{0},{ }_{a c}$ be such that $E_{0}\left(I_{k}\right) u=u$ and $d\left(E_{0}(\lambda) u, u\right) / d \lambda \leqq m^{2}$. We note that such $u$ with finite $m^{2}$ form a dense subset of $E_{0}\left(I_{k}\right) \mathfrak{S}_{0, a c}=$ $E_{0}\left(I_{k}\right) P_{0} \mathfrak{g}$ (see a similar proposition in loc. cit. when $I_{k}$ is the whole interval). If we set $x=\exp \left(-i s \phi\left(H_{0}\right)\right) u$, we have $\left(E_{0}(\lambda) x, x\right)=\left(E_{0}(\lambda) u, u\right)$ so that the assumptions on $x$ stated above are satisfied. Hence (2.1) gives

$$
\begin{aligned}
& \left\|\left(W_{+}-1\right) \exp \left(-i s \phi\left(H_{0}\right)\right) u\right\| \leqq\left(8 \pi m^{2}\|V\|_{1}\right)^{1 / 4} \eta(s)^{1 / 4}, \\
& \eta(s)=\int_{0}^{+\infty}\left\||V|^{1 / 2} \exp \left(-i t H_{0}-i s \phi\left(H_{0}\right)\right) u\right\|^{2} d t \\
& \quad=\sum_{n=1}^{\infty}\left|c_{n}\right| \int_{0}^{+\infty}\left|\left(\exp \left(-i t H_{0}-i s \phi\left(H_{0}\right)\right) u, f_{n}\right)\right|^{2} d t,
\end{aligned}
$$

where $\left\{f_{n}\right\}$ is a complete orthonormal system of eigenvectors of $V$ and the $c_{n}$ are the associated eigenvalues.

The integrals on the right of (2.3) have the form (A1) of Appendix, where $w(\lambda)$ is to be replaced by $d\left(E_{0}(\lambda) u, f_{n}\right) / d \lambda$ which belongs to $L^{2}\left(I_{k}\right)$ with $L^{2}$-norm not exceeding $m$ (see loc. cit.). Therefore, each term on the right of (2.3) tends to 0 for $s \rightarrow+\infty$ (Lemma A3, Appendix). On the other hand, the series on the right of (2.3) is majorized by the convergent series $2 \pi m^{2} \sum\left|c_{n}\right|=2 \pi m^{2}\|V\|_{1}$. Hence $\eta(s) \rightarrow 0$ for $s \rightarrow+\infty$ and the left member of (2.2) must tend to 0 for $s \rightarrow+\infty$. Since $\left(W_{+}-1\right) \exp \left(-i t \phi\left(H_{0}\right)\right)$ is uniformly bounded and the set of $u$ 
with the above properties is dense in $E_{0}\left(I_{k}\right) P_{0} \mathfrak{S}$ as remarked above, it follows that $\left(W_{+}-1\right) \exp \left(-i s \phi\left(H_{0}\right)\right) P_{0} E_{0}\left(I_{k}\right) \rightarrow 0$ strongly for $s \rightarrow+\infty$. But we have $W_{+} \exp \left(-i s \phi\left(H_{0}\right)\right)=\exp \left(-i s \phi\left(H_{1}\right)\right) W_{+}$by (1.2). On multiplying the above result from the left with $\exp \left(i s \phi\left(H_{1}\right)\right)$, we thus obtain

$$
\begin{aligned}
s & -\lim _{s \rightarrow+\infty} \exp \left(i s \phi\left(H_{1}\right)\right) \exp \left(-i s \phi\left(H_{0}\right)\right) P_{0} E_{0}\left(I_{k}\right) \\
& =W_{+} P_{0} E_{0}\left(I_{k}\right)=W_{+} E_{0}\left(I_{k}\right) \quad \text { if } \phi \text { is increasing on } I_{k} .
\end{aligned}
$$

Similarly we can show that

$$
s-\lim _{s \rightarrow+\infty} \exp \left(i s \phi\left(H_{1}\right)\right) \exp \left(-i s \phi\left(H_{0}\right)\right) P_{0} E_{0}\left(I_{k}\right)=W_{-} E_{0}\left(I_{k}\right)
$$

if $\phi$ is decreasing on $I_{k}$.

Since $P_{0} E_{0}(\lambda)$ is continuous in $\lambda$, we have $\sum_{k} P_{0} E_{0}\left(I_{k}\right)=P_{0}$. Adding $(2.4)$ or $\left(2.4^{\prime}\right)$ for $k=1, \cdots, r$, we thus arrive at the result

$$
s-\lim _{s \rightarrow+\infty} \exp \left(i s \phi\left(H_{1}\right)\right) \exp \left(-i s \phi\left(H_{0}\right)\right) P_{0}=\sum_{k=1}^{r} W_{( \pm)} E_{0}\left(I_{k}\right),
$$

where $W_{( \pm)}$means that $W_{+}\left(W_{-}\right)$should be taken if $\phi$ is increasing (decreasing) on $I_{k}$.

(2.5) shows that the wave operator $W_{+}\left(\phi\left(H_{1}\right), \phi\left(H_{0}\right)\right)$ exists and is equal to the right member; it should be noted that the absolutely continuous subspace for $\phi\left(H_{0}\right)$ is identical with $\mathfrak{S}_{0, a c}=P_{0} \mathfrak{E}$ (Lemma A5, Appendix). Similar results hold for $W_{-}\left(\phi\left(H_{1}\right), \phi\left(H_{0}\right)\right)$; we have only to take the opposite choice for $W_{( \pm)}$in (2.5). These wave operators are complete since they also exist when $H_{0}$ and $H_{1}$ are exchanged.

3. Generalization. Let us consider a question which is in a sense converse to Theorem 1. Suppose $\psi\left(H_{1}\right)-\psi\left(H_{0}\right)$ belongs to $\boldsymbol{T}$ for some function $\psi$; then do the wave operators $W_{ \pm}\left(H_{1}, H_{0}\right)$ exist?

The answer to this question is quite simple if $\psi$ is of class $(M)$ and, in addition, univalent. Then the inverse function exists, with domain $\Delta$ consisting of a finite number of open intervals and a finite number of points. This inverse function can be extended to a function $\hat{\psi}$ of class $(M)$ by setting, for example, $\hat{\psi}(\lambda)=\lambda$ on the complement of $\Delta$. Therefore, $\left.W_{ \pm}\left(H_{1}, H_{0}\right)=W_{ \pm}\left(\hat{\psi}\left(\psi\left(H_{1}\right)\right), \hat{\psi}\left(H_{0}\right)\right)\right)$ exist and are complete by Theorem 1.

If $\psi$ is not univalent, we do not know whether the same resalts hold. But we can show that this is true if there is an approximate univalent sequence $\left\{\psi_{n}\right\}$ of functions of class $(M)$ such that $\psi_{n}\left(H_{1}\right)-$ $\psi_{n}\left(H_{0}\right) \in \boldsymbol{T}$. We call $\left\{\psi_{n}\right\}$ an approximate univalent sequence if $\psi_{n}$ is univalent on $(-n, n), n=1,2, \cdots$

More generally, we can prove 
THeOREM 2. Let $H_{0}, H_{1}$ be selfadjoint and let there exist an approximate univalent sequence $\left\{\psi_{n}\right\}$ of functions of class $(M)$ such that $\psi_{n}\left(H_{1}\right)=\psi_{n}\left(H_{0}\right)+V_{n}$ with $V_{n} \in \boldsymbol{T}, n=1,2, \cdots$ Then, for any $\phi \in(M)$, the wave operators $W^{\prime}{ }_{ \pm}=W_{ \pm}\left(\phi\left(H_{1}\right), \phi\left(H_{0}\right)\right)$ exist and are complete. In particular, $W_{ \pm}=W_{ \pm}\left(H_{1}, H_{0}\right)$ exist and are complete. $W^{\prime}{ }_{ \pm}$are piecewise equal either to $W_{ \pm}$or to $W_{\mp}$ in the sense stated in Theorem 1.

Proof. I. The restriction of $\psi_{n}$ to $(-n, n)$ has inverse function, which can be extended to a $\hat{\psi}_{n} \in(M)$ in the same way as above.

Set $\phi_{n}=\phi \circ \hat{\psi}_{n} \circ \psi_{n}$; then $\phi_{n}(\lambda)=\phi(\lambda)$ for $\lambda \in(-n, n)$, and $\phi_{n} \in(M)$ by Lemma A4 (Appendix). We define the following selfadjoint operators, all functions of $H_{j}, j=0,1$ :

$$
\begin{aligned}
\psi_{n}\left(H_{j}\right) & =L_{n j}, & \left(\hat{\psi}_{n} \circ \psi_{n}\right)\left(H_{j}\right) & =H_{n j}, \\
\phi_{n}\left(H_{j}\right)=K_{n j} & =\int \lambda d F_{n j}(\lambda), & \phi\left(H_{j}\right)=K_{j} & =\int \lambda d F_{j}(\lambda) .
\end{aligned}
$$

Since $K_{n j}=\left(\phi \circ \hat{\psi}_{n}\right)\left(L_{n j}\right)$ by operational calculus (see Stone [8], Theorem 6.9), where $\phi \circ \hat{\psi}_{n} \in(M)$ and $L_{n 1}=L_{n 0}+V_{n}, V_{n} \in \boldsymbol{T}$, it follows from Theorem 1 that $W_{n \pm}^{\prime}=W_{ \pm}\left(K_{n 1}, K_{n 0}\right)$ exist and are complete.

II. For any function $\psi$ of class $(M), \psi( \pm \infty)=\lim _{\lambda \rightarrow \pm \infty} \psi(\lambda)$ exist (the values $\pm \infty$ being permitted for these limits). Thus $\phi_{n}( \pm \infty)$ and $\left(\hat{\psi}_{n} \circ \psi_{n}\right)( \pm \infty)$ exist. By replacing $\left\{\phi_{n}\right\}$ by a suitable subsequence (and correspondingly for $\left\{\psi_{n}\right\}$ and $\left.\left\{\hat{\psi}_{n}\right\}\right)$, we may assume that $\alpha_{ \pm} \lim _{n \rightarrow \infty} \phi_{n}( \pm \infty)$ and $\beta_{ \pm}=\lim _{n \rightarrow \infty}\left(\hat{\psi}_{n} \circ \psi_{n}\right)( \pm \infty)$ exist $( \pm \infty$ being permitted for these limits).

Let $J$ be an open interval such that $\alpha_{ \pm}$and $\phi( \pm \infty)$ are exterior to $J$, and let $S=\phi^{-1}(J), S_{n}=\phi_{n}{ }^{-1}(J) . \quad S$ and $S_{n}$ are unions of a finite number of open intervals and of points. Since $K_{j} \phi\left(H_{j}\right)$ and $K_{n_{j}}=$ $\phi_{n}\left(H_{j}\right)$, we have (we denote by $E_{j}(S)$ the spectral measure determined from $\left.\left\{E_{j}(\lambda)\right\}\right)$

$$
F_{j}(J)=E_{j}(S), \quad F_{n j}(J)=E_{j}\left(S_{n}\right), \quad j=0,1 .
$$

$S$ is bounded since $\phi( \pm \infty)$ are exterior to $J$. Similarly, $S_{n}$ is bounded if $n$ is sufficiently large, since $\alpha_{ \pm}$are exterior to $J$.

Take an $n$ so large that $S_{n}$ is bounded and $S \subset(-n, n)$. Since $\phi_{n}(\lambda)=\phi(\lambda)$ for $\lambda \in(-n, n)$, we have $S=(-n, n) \cap S_{n}$. Further take an $m>n$ such that $S_{n} \subset(-m, m)$. We have $S=(-m, m) \cap S_{m}$ as above, so that $S_{m} \cap S_{n}=S_{m} \cap(-m, m) \cap S_{n}=S \cap S_{n}=S$. Hence

$$
\begin{aligned}
F_{n j}(J) F_{m j}(J) & =F_{j}\left(S_{n}\right) E_{j}\left(S_{m}\right) \\
& =E_{j}\left(S_{n} \cap S_{m}\right)=E_{j}(S)=F_{j}(J) .
\end{aligned}
$$

III. Now we have, for any $u \in \mathfrak{S}_{0, a c}=P_{0} \mathfrak{S}$, 


$$
\begin{aligned}
& \exp \left(i t K_{n 1}\right)\left(1-F_{n 1}(J)\right) \exp \left(-i t K_{n 0}\right) P_{0} F_{0}(J) \\
& \quad=\left(1-F_{n 1}(J)\right) \exp \left(i t K_{n 1}\right) \exp \left(-i t K_{n 0}\right) P_{0} F_{0}(J) \\
& \rightarrow\left(1-F_{n 1}(J)\right) W^{\prime}{ }_{n+} F_{0}(J) \quad \text { strongly for } t \rightarrow+\infty .
\end{aligned}
$$

Since $\left(1-F_{n 1}(J)\right) W^{\prime}{ }_{n+}=W^{\prime}{ }_{n+}\left(1-F_{n 0}(J)\right)$ by (1.2) applied to $W^{\prime}{ }_{n+}$, and since $F_{0}(J) \leqq F_{n 0}(J)$ by (3.3), the last member of (3.4) vanishes. On the other hand $\exp \left(-i t K_{n 0}\right) F_{0}(J)=\exp \left(-i t K_{0}\right) F_{0}(J)$ since $\phi_{n}(\lambda)=$ $\phi(\lambda)$ for $\lambda \in(-n, n)$ and $F_{0}(J)=E_{0}(S) \leqq E_{0}((-n, n))$. On multiplying (3.4) from the left by $\exp \left(-i t K_{n 1}\right)$, we thus obtain

$$
s-\lim _{t \rightarrow+\infty}\left(1-F_{n 1}(J)\right) \exp \left(-i t K_{0}\right) P_{0} F_{0}(J)=0 .
$$

The same is true when $n$ is replaced by the $m>n$ considered above. Now multiply the latter from the left by $F_{n 1}(J)$ and add to (3.5). In view of (3.3), we then obtain

$$
s-\lim _{t \rightarrow+\infty}\left(1-F_{1}(J)\right) \exp \left(-i t K_{0}\right) P_{0} F_{0}(J)=0 .
$$

Multiply again (3.6) from the left by $\exp \left(i t K_{1}\right)$; then

$$
\begin{aligned}
s & -\lim _{t \rightarrow+\infty} \exp \left(i t K_{1}\right) \exp \left(-i t K_{0}\right) P_{0} F_{0}(J) \\
& =s-\lim _{t \rightarrow+\infty} F_{1}(J) \exp \left(i t K_{n 1}\right) \exp \left(-i t K_{n 0}\right) P_{0} F_{0}(J) \\
& =F_{1}(J) W_{n+}^{\prime} F_{0}(J),
\end{aligned}
$$

where we have again used the relation

$$
\exp \left(-i t K_{0}\right) F_{0}(J)=\exp \left(-i t K_{n 0}\right) F_{0}(J)
$$

and similarly $\exp \left(i t K_{1}\right) F_{1}(J)=\exp \left(i t K_{n 1}\right) F_{1}(J)=F_{1}(J) \exp \left(i t K_{n 1}\right)$.

(3.7) shows that $\lim _{t \rightarrow+\infty} \exp \left(i t K_{1}\right) \exp \left(-i t K_{0}\right) u$ exists and is equal to $\left.F_{1}(J) W^{\prime}{ }_{n}\right\lrcorner u$ whenever $u$ belongs to $P_{0} F_{0}(J) \mathscr{S}$, where $J$ is any interval with the four points $\alpha_{ \pm}$and $\phi( \pm \infty)$ in its exterior. Since such $u$ forms a dense set in $P_{0} \mathfrak{E}$, the existence of $W_{+}^{\prime}=W_{+}\left(K_{1}, K_{0}\right)$ has been proved. The existence of $W^{\prime}$ - can be proved in the same way. Since $K_{0}$ and $K_{1}$ can be exchanged, all these wave operators are complete.

Incidentally, it follows from (3.7) that $W^{\prime}{ }_{+} u=F_{1}(J) W^{\prime}{ }_{n+} u$ for $u \in P_{0} F_{0}(J) \mathscr{S}_{\text {. }}$ But $\left\|W^{\prime}{ }_{+} u\right\|=\|u\|=\left\|W^{\prime}{ }_{n+} u\right\|$ since $W^{\prime}{ }_{+}$and $W_{{ }_{+n}}$ are isometric on $P_{0} \mathfrak{S}$. Since $F_{1}(J)$ is a projection, we must have $W^{\prime}{ }_{+} u=W^{\prime}{ }_{n+} u$. Similar result holds for $W^{\prime}$. Thus

$$
\left(W^{\prime}{ }_{ \pm}-W^{\prime}{ }_{n \pm}\right) F_{0}(J)=0 \text {. }
$$

Note that this is true for sufficiently large $n$ (depending on $J$ ).

IV. To prove the piecewise equality of ${W^{\prime}}_{ \pm}$and $W_{ \pm}$or $W_{\mp}$, let $I_{k}$ be one of the intervals associated with $\phi \in(M)$. We may assume 
that $\phi^{\prime}>0$ on $I_{k}$; we have to show that $\left(W^{\prime}{ }_{ \pm}-W_{ \pm}\right) E_{0}\left(I_{k}\right)=0$. For this it suffices to show that $\left(W^{\prime}{ }_{ \pm}-W_{ \pm}\right) E_{0}(I)=0$ for any finite subinterval $I$ of $I_{k}$; we may further assume that $\beta_{ \pm}$are exterior to $I$ and $\alpha_{ \pm}, \phi( \pm \infty)$ are exterior to the interval $\phi(I)$.

We set $J=\phi(I)$ and apply the preceding results to $J$. Since $S=\phi^{-1}(J) \supset I$, we have $E_{j}(I) \leqq E_{j}(S)=F_{j}(J)$ and hence by (3.8)

$$
\left(W^{\prime}{ }_{ \pm}-W^{\prime}{ }_{n \pm}\right) E_{0}(I)=0
$$

for sufficiently larg $n$.

We have similar results when $\phi(\lambda)$ is replaced by the identity function $\lambda$ (since $\beta_{ \pm}$and $\pm \infty$ are exterior to $I$ ). Then $W_{ \pm}^{\prime}, W_{n \pm}^{\prime}$ are to be replaced respectively by $W_{ \pm}=W_{ \pm}\left(H_{1}, H_{0}\right)$ and $W_{n \pm}=W_{ \pm}\left(H_{n 1}, H_{n 0}\right)$. Thus

$$
\left(W_{ \pm}-W_{n \pm}\right) E_{0}(I)=0
$$

for sufficiently large $n$.

We may assume that $n$ is so large that $I \subset(-n, n)$. $I$ can be expressed as the union of a finite number of subintervals $\Delta_{p}$ (and a finite number of points) in each of which $\psi_{n}$ is monotonic. Then $\hat{\psi}_{n}$ is monotonic on $\Delta^{\prime}{ }_{p}=\psi_{n}\left(\Delta_{p}\right)$ since $\psi_{n}$ is univalent on $(-n, n)$. $\phi \circ \hat{\psi}_{n}$ is also monotonic on $\Delta^{\prime}{ }_{p}$ since $\phi^{\prime}>0$ on $\hat{\psi}_{n}\left(\Delta^{\prime}{ }_{p}\right)=A_{p}$; it is increasing or decreasing with $\hat{\psi}_{n}$. Since $K_{n j}=\left(\phi \circ \hat{\psi}_{n}\right)\left(L_{n j}\right), H_{n j}=\hat{\psi}_{n}\left(L_{n j}\right)$ and $L_{n 1}=L_{n 0}+V_{n}, V_{n} \in \boldsymbol{T}$, it follows from Theorem 1 that $\left(W_{n \pm}^{\prime}-\right.$ $\left.W_{n \pm}\right) E_{0}\left(\Delta_{p}\right)=0$; note that $E_{0}\left(\Delta_{p}\right) \leqq E_{0}\left(\psi_{n}^{-1}\left(\Delta^{\prime}{ }_{p}\right)\right)=G_{0}\left(\Delta_{p}^{\prime}\right)$ where $\left\{G_{0}(\lambda)\right\}$ is the resolution of the identity for $L_{n 0}=\psi_{n}\left(H_{0}\right)$. Adding the results obtained for $p=1,2, \cdots$, we have

$$
\left(W_{n \pm}^{\prime}-W_{n \pm}\right) E_{0}(I)=0 \text {. }
$$

The desired result $\left(W^{\prime}{ }_{ \pm}-W_{ \pm}\right) E_{0}(I)=0$ follows from (3.9), (3.10) and (3.11).

4. Applications. A number of sufficient conditions for the existence and completeness of wave operators can be deduced from Theorem 1 or 2 . We shall mention only a few.

(a) Let neither $H_{0}$ nor $H_{1}$ have the eigenvalue 0 . If $H_{1}^{-p}=H_{0}^{-p}+V$ with $V \in \boldsymbol{T}$ for some odd integer $p$, then $W_{ \pm}\left(\phi\left(H_{1}\right), \phi\left(H_{0}\right)\right)$ exist and are complete for any $\phi \in(M)$.

The proof follows by applying Theorem 2 with $\psi_{n}=\psi$ (independent of $n$ ) where $\psi(\lambda)=\lambda^{-p}$ for $\lambda \neq 0$ and $\psi(0)=0$.

(b) In (a) we may allow even integers $p$ if we assume in addition 
that $H_{0}$ and $H_{1}$ are nonnegative.

In this case we need only to replace the above $\psi$ by $\psi(\lambda)=$ $(\operatorname{sign} \lambda)|\lambda|^{-p}$ for $\lambda \neq 0$.

(c) Let $\left(H_{1}-\zeta\right)^{-1}-\left(H_{0}-\zeta\right)^{-1} \in \boldsymbol{T}$ for some nonreal complex number $\zeta$. Then $W_{ \pm}\left(\phi\left(H_{1}\right), \phi\left(H_{0}\right)\right)$ exist and are complete for any $\phi \in(M)$.

For the proof we first note that, if the assumption is true for some $\zeta=\zeta_{0}$, then it is true also for all nonreal $\zeta$. This can be seen first for $\left|\zeta-\zeta_{0}\right|<\left|\operatorname{Im} \zeta_{0}\right|$ by considering the Neumann series for the resolvents. The result can then be extended to all $\zeta$ of the half-plane $(\operatorname{Im} \zeta)\left(\operatorname{Im} \zeta_{0}\right)>0$ by a standard procedure. The other half-plane can be taken care of by considering the adjoints.

Set now $\psi_{n}(\lambda)=-i\left[(n-i \lambda)^{-1}-(n+i \lambda)^{-1}\right]=2 \lambda\left(n^{2}+\lambda^{2}\right)^{-1}$. It follows from the above remark that $\psi_{n}\left(H_{1}\right)-\psi_{n}\left(H_{0}\right) \in \boldsymbol{T}$. But it is easy to see that $\left\{\psi_{n}\right\}$ is an approximate univalent sequence of functions of class $(M)$. Hence the proposition follows by Theorem 2 .

(b) It should be remarked that the existence of $W_{ \pm}\left(\phi\left(H_{1}\right), \phi\left(H_{0}\right)\right)$ implies the existence of

$$
s-\lim _{n \rightarrow \pm \infty} U_{1}^{n} U_{0}^{-n}=W_{ \pm}\left(H_{1}, H_{0}\right),
$$

where $U_{j}=\left(H_{j}-i\right)\left(H_{j}+i\right)^{-1}$ is the Cayley transform of $H_{j}$. In fact, $U_{j}=\exp \left(i \phi\left(H_{j}\right)\right)$ where $\phi(\lambda)=-2 \operatorname{arccot} \lambda$, and $\phi$ belongs to $(M)$, being strictly increasing on $(-\infty,+\infty)$.

Appendix. We prove here some lemmas which are used in the text.

LEMMA A1. Let $f, g$ be complex-valued, continuous functions on a closed interval $[a, b]$. Let $f$ be of bounded variation with total variation $V_{f}$. Let $G(\lambda)=\int_{a}^{\lambda} g(\lambda) d \lambda$ and let $M_{G}=\max |G(\lambda)|, M_{f}=$ $\max |f(\lambda)|$. Then $\left|\int_{a}^{b} f(\lambda) g(\lambda) d \lambda\right| \leqq\left(M_{f}+V_{f}\right) M_{G}$.

The proof is simple and will be omitted.

Lemma A2. Let $\phi$ be a real-valued differentiable function on $[a, b]$ such that the derivative $\phi^{\prime}$ is continuous, positive and of bounded variation. We have for any $t, s>0$

$$
\left|\int_{a}^{b} \exp (i t \lambda-i s \phi(\lambda)) d \lambda\right| \leqq \frac{2\left(c+V_{\phi^{\prime}}\right)}{c(t+c s)}
$$

where $c=\min \phi^{\prime}(\lambda)>0$ and $V_{\phi^{\prime}}$ is the total variation of $\phi^{\prime}$. 
Proof. The integral in question is equal to

$$
\int_{a}^{b} i\left(t+s \phi^{\prime}(\lambda)\right)^{-1}(d / d \lambda) \exp (-i t \lambda-i s \phi(\lambda)) d \lambda
$$

We apply Lemma A1 to estimate this integral, setting $f(\lambda)=$ $i\left(t+s \phi^{\prime}(\lambda)\right)^{-1}$ and $g(\lambda)=(d / d \lambda) \exp (-i t \lambda-i s \phi(\lambda))$. Then $M_{f}=(t+c s)^{-1}$, $M_{G} \leqq 2$ and it is easily seen that $V_{f} \leqq s V_{\phi^{\prime}} /(t+c s)^{2} \leqq V_{\phi^{\prime}} / c(t+c s)$. This proves the desired inequality.

LEMMA A3. Let $\phi$ be of class $(M)$ with an associated system of of intervals $\left\{I_{k}\right\}$ (see definition in text). For a fixed $k$, let $w \in L^{2}\left(I_{k}\right)$. If $\phi$ is increasing on $I_{k}$, we have

$$
\int_{0}^{+\infty} d t\left|\int_{-\infty}^{+\infty} \exp (-i t \lambda-i s \phi(\lambda)) w(\lambda) d \lambda\right|^{2} \longrightarrow 0, \quad s \rightarrow+\infty .
$$

If $\phi$ is decreasing on $I_{k^{\prime}}$ (A1) is true if $\int_{0}^{+\infty} d t$ is relpaced by $\int_{-\infty}^{0} d t$.

Proof. We may assume that $w \in L^{2}(-\infty,+\infty)$, on setting $w(\lambda)=$ 0 for $\lambda$ outside $I_{k}$. Let $H$ be the selfadjoint operator $H u(\lambda)=\lambda u(\lambda)$ acting in $L^{2}(-\infty,+\infty)$, and let $U$ be the unitary operator defined by the Fourier transformation. The inner integral of (A1) represents the function $(U \exp (-i s \phi(H)) w(t)$, and the left member of (A1) is equal to $\|E U \exp (-i s \phi(H)) w\|^{2}$, where $E$ is the projection of $L^{2}(-\infty$, $+\infty)$ onto the subspace consisting of all functions that vanish on $(-\infty, 0)$. Thus (Al) is equivalent to that $E U \exp (-i s \phi(H)) w \rightarrow 0$, $s \rightarrow+\infty$. Since $E U \exp (-i s \phi(H))$ is uniformly bounded with norm $\leqq 1$, it suffices to prove (A1) for all $w$ belonging to a fundamental subset of $L^{2}\left(I_{k}\right)$. Thus we may restrict ourselves to considering only characteristic functions $w$ of closed finite subintervals $[a, b]$ of $I_{k}$ 。

Assume that $\phi$ is increasing on $I_{k}$. If we denote by $v_{s}(t)$ the inner integral of (A1) for the characteristic function $w$ of $[a, b] \subset I_{k}$, we have by Lemma A2

$$
\left|v_{s}(t)\right| \leqq \frac{2\left(c+V_{\phi^{\prime}}\right)}{c(t+c s)} \text { so that } \int_{0}^{+\infty}\left|v_{s}(t)\right|^{2} d t \leqq \frac{4\left(c+V_{\phi^{\prime}}\right)^{2}}{c^{3} s} \longrightarrow 0
$$

for $s \rightarrow+\infty$, where $c$ is the minimum of $\phi^{\prime}(\lambda)$ on $[a, b]$ and $V_{\phi^{\prime}}$ is the total variation of $\phi^{\prime}$ on $[a, b]$. A similar proof applies to the case $\phi^{\prime}<0$ on $I_{k}$, with $\int_{0}^{+\infty} d t$ replaced by $\int_{-\infty}^{0} d t$.

Lemma A4. Let $\phi, \psi$ be of class $(M)$. Then the composed function $\phi \circ \psi$ also belongs to $(M)$, and there exists a system of intervals associated with $\phi \circ \psi$ such that, in each interval of the system, both $\psi$ and $\phi \circ \psi$ are monotonic. 
Proof. Let $\left\{I_{k}\right\}$ and $\left\{J_{h}\right\}$ be systems of intervals associated with $\phi$ and $\psi$, respectively. For each $h, \psi$ maps $J_{h}$ one-to-one onto an open interval $J^{\prime}{ }_{h}$. Let $J_{k h}$ be the inverse image under this map of $J^{\prime}{ }_{h} \cap h_{k}$. Obviously all $J_{k h}$ are open and mutually disjoint, and cover the whole interval $(-\infty,+\infty)$ except for a finite number of points. It is easy to see that $\phi \circ \psi$ is monotonic and continuously differentiable on each $J_{k n}$, with $(\phi \circ \psi)^{\prime}(\lambda)=\phi^{\prime}(\psi(\lambda)) \psi^{\prime}(\lambda)$. Furthermore, $(\phi \circ \psi)^{\prime}$ is locally of bounded variation on $J_{k n}$, for the same is true with $\phi^{\prime}$ and $\psi^{\prime}$ by assumption. The intervals $J_{k n}$ form a system stated in the lemma.

Lemma A5. Let $\phi$ be of class $(M)$. For any selfadjoint operator $H$, the absolutely continuous subspace for $\phi(H)$ is identical with the absolutely continuous subspace for $H$.

Proof. Let $H=\int \lambda d E(\lambda), \phi(H)=\int \lambda d F(\lambda)$ be the spectral representations of the operators considered. We denote by $E(S), F(S)$ the spectral measures constructed from $\{E(\lambda)\},\{F(\lambda)\}$, respectively. For any Borel subsets $S$ of the real line, we have $F(S)=E\left(\phi^{-1}(S)\right)$. If $|S|=0$ (we denote by $|S|$ the Lebesgue measure of $S$ ), then $\left|\phi^{-1}(S)\right|=0$ by the properties of $\phi \in(M)$, so that $F(S) u=0$ if $u$ is absolutely continuous with respect to $H$. On the other hand, $F(\phi(S))=E\left(\phi^{-1}(\phi(S)) \geqq\right.$ $E(S)$. If $|S|=0$, we have $|\phi(S)|=0$ so that $\|E(S) u\| \leqq\|F(\phi(S)) u\|=0$ if $u$ is absolutely continuous with respect to $\phi(H)$. This proves the lemma.

\section{BIBLIOGRAPHY}

1. M. Sh. Birman, On the conditions for the existence of wave operators, Doklady Akad. Nauk USSR 143 (1962), 506-509.

2. - A test for the existence of wave operators, Doklady Akad. Nauk USSR 147 (1962), 1008-1009.

3. M. Sh. Birman, and M.G. Krein, On the theory of wave and scattering operators, Doklady Akad. Nauk USSR 144 (1962), 475-478.

4. T. Kato, On finite-dimensional perturbations of self-adjoint operators, J. Math. Soc. Japan 9 (1957), 239-249.

5. — Perturbation of continuous spectra by trace class operators, Proc. Japan Acad. 33 (1957), 260-264.

6. S. T. Kuroda, On the existence and the unitary property of the scattering operators, Nuovo Cimento 12 (1959), 431-454.

7. - Perturbation of continuous spectra by unbounded operators, I and II, J. Math. Soc. Japan 11 (1959), 247-262; 12 (1960), 243-257.

8. M. H. Stone, Linear transformations in Hilbert space and their applications to analysis, AMS Colloq. Publ., 1932. 


\section{PACIFIC JOURNAL OF MATHEMATICS}

\section{EDITORS}

H. Samelson

Stanford University

Stanford, California

R. M. Blumenthal

University of Washington

Seattle, Washington 98105
J. Dugundu

University of Southern California Los Angeles, California 90007

*Richard Arens

University of California

Los Angeles, California 90024

\section{ASSOCIATE EDITORS}
E. F. BECKENBACH
B. H. NeumanN
F. WOLF
K. YOSIDA

\section{SUPPORTING INSTITUTIONS}

UNIVERSITY OF BRITISH COLUMBIA CALIFORNIA INSTITUTE OF TECHNOLOGY UNIVERSITY OF CALIFORNIA MONTANA STATE UNIVERSITY

UNIVERSITY OF NEVADA

NEW MEXICO STATE UNIVERSITY

OREGON STATE UNIVERSITY

UNIVERSITY OF OREGON

OSAKA UNIVERSITY

UNIVERSITY OF SOUTHERN CALIFORNIA
STANFORD UNIVERSITY

UNIVERSITY OF TOKYO

UNIVERSITY OF UTAH

WASHINGTON STATE UNIVERSITY

UNIVERSITY OF WASHINGTON

AMERICAN MATHEMATICAL SOCIETY CALIFORNIA RESEARCH CORPORATION SPACE TECHNOLOGY LABORATORIES NAVAL ORDNANCE TEST STATION 


\section{Pacific Journal of Mathematics}

\section{Vol. 15, No. $1 \quad$ September, 1965}

Donald Charles Benson, Unimodular solutions of infinite systems of linear

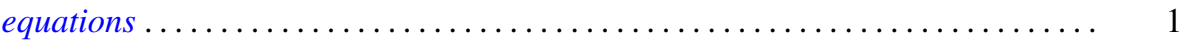

Richard Earl Block, Transitive groups of collineations on certain designs . . . . . . 13

Barry William Boehm, Existence of best rational Tchebycheff approximations .... . 19

Joseph Patrick Brannen, A note on Hausdorff's summation methods . . . . . . . . . . 29

Dennison Robert Brown, Topological semilattices on the two-cell ............ 35

Peter Southcott Bullen, Some inequalities for symmetric means . . . . . . . . . . 47

David Geoffrey Cantor, On arithmetic properties of coefficients of rational

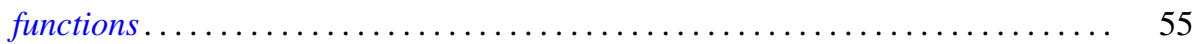

Luther Elic Claborn, Dedekind domains and rings of quotients . . . . . . . . . 59

Allan Clark, Homotopy commutativity and the Moore spectral sequence ........ 65

Allen Devinatz, The asymptotic nature of the solutions of certain linear systems of

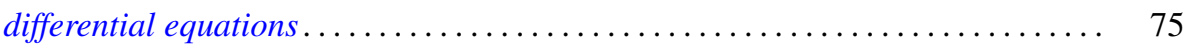

Robert E. Edwards, Approximation by convolutions ................... 85

Theodore William Gamelin, Decomposition theorems for Fredholm operators . . . . . 97

Edmond E. Granirer, On the invariant mean on topological semigroups and on

topological groups .................................. 107

Noel Justin Hicks, Closed vector fields . . . . . . . . . . . . . . . . . . . 141

Charles Ray Hobby and Ronald Pyke, Doubly stochastic operators obtained from

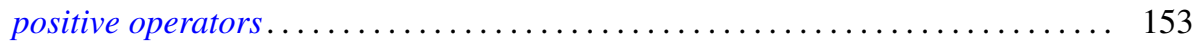

Robert Franklin Jolly, Concerning periodic subadditive functions . . . . . . . . . 159

Tosio Kato, Wave operators and unitary equivalence . . . . . . . . . . . . . . 171

Paul Katz and Ernst Gabor Straus, Infinite sums in algebraic structures . . . . . . . 181

Herbert Frederick Kreimer, Jr., On an extension of the Picard-Vessiot theory ...... 191

Radha Govinda Laha and Eugene Lukacs, On a linear form whose distribution is

identical with that of a monomial ......................... 207

Donald A. Ludwig, Singularities of superpositions of distributions . . . . . . . . . 215

Albert W. Marshall and Ingram Olkin, Norms and inequalities for condition

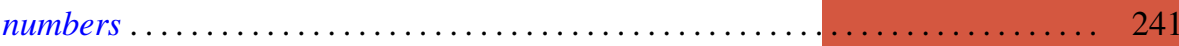

Horace Yomishi Mochizuki, Finitistic global dimension for rings . . . . . . . . . . 249

Robert Harvey Oehmke and Reuben Sandler, The collineation groups of division

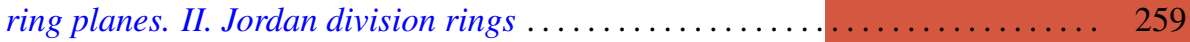

George H. Orland, On non-convex polyhedral surfaces in $E^{3} \ldots \ldots \ldots \ldots \ldots \ldots \ldots 267$

Theodore G. Ostrom, Collineation groups of semi-translation planes . . . . . . . . 273

Arthur Argyle Sagle, On anti-commutative algebras and general Lie triple

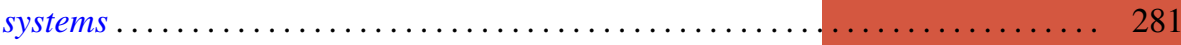

Laurent Siebenmann, A characterization of free projective planes . . . . . . . . . 293

Edward Silverman, Simple areas.................................. 299

James McLean Sloss, Chebyshev approximation to zero .................. 305

Robert S. Strichartz, Isometric isomorphisms of measure algebras . . . . . . . . . 315

Richard Joseph Turyn, Character sums and difference sets . . . . . . . . . . . . 319

L. E. Ward, Concerning Koch's theorem on the existence of arcs . . . . . . . . . . 347

Israel Zuckerman, A new measure of a partial differential field extension ......... 357 